doi:10.1017/\$1041610219000097

\section{Translating knowledge into practice at the local level: evaluation of a Pilot CME for primary care physicians on dementia early diagnosis and management}

Over 30 countries have adopted National Strategic Plans for Dementia care (NSPD) (Chow et al., 2018). These strategies are considered the single most powerful means to transform dementia care at the national level (Alzheimer's Disease International, 2017). Although NSPD are unique to each country, they share some objectives, for example, improving training and education of professionals working with people with dementia (PwD) is a priority in the NSPDs' of 16 countries. In some countries, the NSPD have led to greater emphasis on early diagnosis. However, there are challenges in the implementation of the nationally accepted diagnostic and management guidelines (Chow et al., 2018).

As a common first point of contact for patients dealing with cognitive decline, primary care physicians (PCP) play a key role in timely diagnosis and management of dementia. PCPs around the world, have expressed an interest for further education and training, yet the best method to accomplish this objective has yet to be determined (Schütze et al., 2018). Global and national guidelines need to be translated into practical and locally relevant training programs in order to have an effect on practices. This is especially true for dementia where local social services and non-pharmacological interventions need to be coordinated early in the course of the disease (Cohen-Mansfield, 2018). Most PCPs training programs reported in the literature, focus on national or international workshops, which although possibly effective in terms of knowledge transfer, do not create local connections among the available social and medical systems, resources and experts that may allow not only transfer of knowledge to PCPs, but also facilitate changes in practices and sustainability at the local level (Chow et al., 2018; Schütze et al., 2018).

We report on the development and evaluation of a pilot CME for PCPs. The CME course aimed to: impact on the knowledge, attitudes, confidence and practices of PCPs in relation to diagnosis and treatment of people with dementia (PwD) and their family caregivers. Our vision was to facilitate local community-based social services and medical experts to address the Israeli NSPD guidelines (Lemberger et al., 2017).

MELABEV is a community-based NGO serving $\mathrm{PwD}$ and their families from diagnosis to end of life, for over 30 years. Services include four daycare centers, homecare, and family support. The idea for a training program for PCP's came in part from MELABEV staff who noticed that clients are referred to MELABEV rather late in the dementia process. MELABEV Research team partnered with the Department of Family Medicine, central District, Clalit Health Services (CHS), the largest health maintenance organization (HMO) in Israel, and the Department of Rehabilitation and Geriatric Medicine of Hadassah-Hebrew University Medical Centers. We developed and implemented a one-day CME workshop on dementia. The following national objectives are reflected in our pilot CME course: improving professional training for dementia care, increasing referrals for non-pharmacological treatment, and strengthening the communication, cooperation and integration between health and social care systems.

The CME was incorporated into the already existent weekly PCPs CME framework and taught by a multidisciplinary local team including topics such as: early diagnosis, assessment tools, pharmacological and non-pharmacological interventions, community support services, and advance directives planning. There was time for questions and answers, including personal interaction with local experts. Topic specific written materials were distributed.

Pre/post self-complete questionnaires were used for CME evaluation. There were 33 PCPs who attended the CME training from 100 potential participants. Twenty-one PCPs returned the pre-intervention questionnaire. Using SPSS version 25 to analyze the answers of 15 PCPs who filled out pre and post CME questionnaires, two separate paired-samples t-test were conducted.

The results revealed significant differences in the number of diagnostic tools that were mentioned by the participants before $(\mathrm{M}=2.00, \mathrm{SD}=1.363)$ and after $(M=3.13, S D=0.389)$ the workshop; $t$ (14) $=-2.552, \mathrm{p}<0.05$.

As well as a significant difference in the number of non-pharmacological interventions that were mentioned by the participants before $(M=2.00$, $\mathrm{SD}=1.464)$ and after $(\mathrm{M}=3.2, \mathrm{SD}=1.656)$ the workshop; $\mathrm{t}(14)=-2.553, \mathrm{p}<0.05$.

Our findings enrich current research about effective dementia educational interventions that improve 
self-reported knowledge, attitudes, confidence and practices of PCPs including topics that are related to available social resources and non-pharmacological services locally available to PCPs. Furthermore, the PCPs were directly exposed to locally available specialists, community-based institutions/resources and professionals that they could connect to for further assistance in real practice scenarios. The course contents were rated as highly valued by the participants and strongly recommended for their colleagues, indicating that the course was well received. We suggest that local-based CME courses is a venue that may impact the transference of knowledge to practice to meet NSPD guidelines at the local level. Similar studies focusing on local experts and integration of social and medical resources relevant to the implementation of national dementia strategies with larger number of PCPs may lead to a new venue of dissemination and sustainability of national strategies on dementia.

\section{Conflict of interest}

None.

\section{Description of authors' roles}

R. Berenbaum designed the study, supervised the data collection and wrote the article. C. Tziraki designed the study and assisted with writing the article. J. Dresner designed the study, and assisted with writing the article. Y. Maaravi assisted with the design of the study and with the writing of the article. $\mathrm{N}$. Pivko assisted with the design of the study. B. Erlich carried out the statistical analysis and assisted with writing the article.

\section{Acknowledgments}

We would like to thank all the PCP who filled in the questionnaires.

\section{References}

Alzheimer's Disease International (2017). Dementia Plans [Internet]. London, UK: Alzheimer's Disease International.

Chow, S., et al. (2018). National dementia strategies: what should Canada learn? Canadian Geriatrics fournal, 21, 173-209. doi: 10.5770/cgj.21.299.

Cohen-Mansfield, J. (2018). Non-pharmacological interventions for persons with dementia: what are they and how should they be studied? International Psychogeriatrics, 30, 281-283. doi: 10.1017/S1041610218 00039X.

Lemberger, J., Rasooly, I., Sternberg, S., Brodsky, J. and Laron, S. (2017) From research to policy, to implementation: the case of the Israeli National Strategic plan to address Alzheimer's and other types of dementia. Alzheimer's $\mathcal{E}$ Dementia, 13, P1468.

Schütze, H.,Shell, A. and Brodaty, H. (2018). Development, implementation and evaluation of Australia's first national continuing medical education program for the timely diagnosis and management of dementia in general practice. BMC Medical Education, 18, 194. doi: 10.1186/ s12909-018-1295-y.

Rakel Berenbaum, ${ }^{1}$ (iD JUlian Dresner, ${ }^{2,3}$ Yoram MaAravi, ${ }^{4}$ Bracha Erlich, ${ }^{5}$ Nechama Pivko ${ }^{6}$ and Chariklia Tziraki ${ }^{1}$

${ }^{1}$ Research and Development Department, Melabev Community Clubs for Eldercare, Jerusalem, Israel

${ }^{2}$ Department of Family Medicine, Central District Clalit Health Services, Department of Family Medicine, Rishon L'zion, Israel Tel Aviv University, Tel Aviv, Israel

${ }^{3}$ Tel Aviv University, Tel Aviv, Israel

${ }^{4}$ Department of Geriatric Medicine and Rehabilitation, Hadassah-Hebrew University Medical Centers, Jerusalem, Israel

${ }^{5}$ Israel Gerontological Data Center, Paul Baerwald School of Social Work and Social Welfare, the Hebrew University of Jerusalem, Jerusalem, Israel

${ }^{6}$ Social Work Department, Melabev - Community Clubs for Eldercare, Jerusalem, Israel

Email: melabev1@gmail.com 